\title{
Evidências científicas de enfermagem sobre idosos estomizados
}

\section{Scientific evidence from nursing on elderly ostomy}

\author{
Amanda Travassos da Costa' • Pedro Paulo Corrêa Santana² • Phelipe Austríaco Teixeira ${ }^{*}$ \\ Fátima Helena do Espírito Santo ${ }^{4}$ Diana Mary Araújo de Melo Flach ${ }^{5}$ Marilda Andrade ${ }^{6}$
}

\begin{abstract}
RESUMO
No processo de envelhecimento, o ser humano passa por diversas mudanças, e dentre as consequências de certas doenças crônicas não transmissíveis junto ao processo de envelhecimento, está a confecção de um estoma. Desta forma, o estudo é norteado pela questão: quais as evidências científicas de enfermagem acerca das pessoas idosas com colostomia, no período de 2005 a 2015? Tendo como objetivo geral levantar publicações científicas de enfermagem sobre pessoas idosas estomizadas no recorte temporal de 2005 a 2015 e como objetivos específicos analisar as principais dificuldades vivenciadas por esses indivíduos, descrevendo as estratégias de enfermagem frente a tais dificuldades. Trata-se de um estudo exploratório, descritivo, realizado por meio de revisão integrativa da literatura. As buscas foram realizadas nas bases de dados LILACS, BDENF, MEDLINE, IBECS e SciELO por meio da associação dos descritores idoso, enfermagem, colostomia. Como resultados tem-se as dificuldades enfrentadas pelos idosos, destacando-se desconhecimento, ansiedade e medo, dentre as estratégias de enfermagem destacouse a educação em saúde. Concluiu-se que as pessoas idosas estomizadas demandam um cuidado que ultrapasse as orientações realizadas em um plano de alta, pois o cuidado a estes necessita ser integral, propiciando abordagens diferenciadas aos diversos contextos nos quais estão inseridos, inclusive no seu âmbito familiar.
\end{abstract}

Palavras-chave: Idoso; Enfermagem; Colostomia; Enfermagem; Gerontologia.

\begin{abstract}
In the aging process, the human being goes through several changes as among the consequences of certain chronic disease with the aging process, it is colostomy. Hence, the study is guided by the question: what is the scientific evidence of Nursing about colostomy elderly from 2005 to 2015? With the overall goal to raise scientific publications on Nursing colostomy in elderly in the time frame 2005-2015 and the specific objectives, to analyze the main difficulties experienced by these individuals, describing the Nursing strategies to the difficulties. It is an exploratory and descriptive study, conducted through an integrative literature review. The searches were conducted in the databases LILACS, BDENF, MEDLINE, SCIELO and IBECS; and through the association of descriptors elderly, nursing and colostomy. The results showed the difficulties faced by older people, especially ignorance, anxiety, fear, and, among the nursing strategies stood out health education. It was concluded that the colostomy elderly require care that exceed the guidelines made in a high level, because the care they need to be full, providing different approaches to the various contexts in which they are inserted, including the family environment.
\end{abstract}

Keywords: Elderly; Colostomy; Nursing; Gerontology; Nursing.

${ }^{1}$ Enfermeira e discente do curso de especialização em enfermagem gerontológica pela Universidade Federal Fluminense. amandatrv@gmail.com

${ }^{2}$ Enfermeiro Gerontólogo e Mestre em Ciências do Cuidado em Saúde pela Escola de Enfermagem Aurora Afonso Costa (EEAAC), Universidade Federal Fluminense (UFF). psantana.uff@gmail.com

${ }^{3}$ Enfermeiro. Especialista em Controle de Infecção pela EEAAC/UFF. Mestre em Medicina Tropical pela FIOCRUZ.ph-austriaco@hotmail.com

${ }^{4}$ Enfermeira. Doutora em enfermagem. Professora Associada da EEAAC/UFF. Coordenadora do curso de Especialização em Enfermagem Gerontológica na EEAAC/UFF. fatahelen@hotmail.com

${ }^{5}$ Enfermeira. Mestre e doutoranda em Ciências do Cuidado em Saúde pela EEAAC/UFF. dflach@superig.com.br

${ }^{6}$ Enfermeira. Doutora em enfermagem. Vice-Diretora e Professora Associada da EEAAC/UFF. marildaandrade@uol.com.br

Os autores declaram que não houve conflitos de interesse na concepção deste trabalho. 
Dentre as transformações ocorridas no decorrer do século XX destaca-se a mudança no perfil de morbidade e mortalidade da população, que resultou em um crescimento total no número de pessoas idosas de $55,3 \%$ em um intervalo de 25 anos - 1980 a $2005^{1}$.

Destaca-se nesses números, a faixa de idade com mais de 80 anos, que apresentou um crescimento de $246 \%$ segundo dados do Instituto Brasileiro de Geografia e Estatística $(\mathrm{IBGE})^{2}$, alterando a composição interna do próprio grupo de pessoas idosas e revelando uma heterogeneidade de características deste segmento populacional, o que vem refletir a ocorrência do que denominamos como transição demográfica acelerada.

As estimativas do IBGE indicam que, no ano de 2025, o Brasil deverá ter um contingente de 34 milhões de idosos, representando $15 \%$ da população total, podendo ocupar o lugar de sexta população idosa do mundo nesta mesma época ${ }^{3}$.

Uma das modificações frequentes durante o processo de envelhecimento é o surgimento das Doenças Crônicas não Transmissíveis (DCNT's), que são doenças multifatoriais de longa duração, desenvolvidas no decorrer da vida, também consideradas um sério problema de saúde pública e atualmente são responsáveis por aproximadamente $63 \%$ das mortes no mundo ${ }^{4}$. Caracterizadas por possuírem fatores de risco em comum e por necessitarem de tratamento contínuo, estão entre elas o câncer, diabetes, doenças do aparelho circulatório e respiratório. Dentre algumas das consequências de certas DCNT's junto ao processo de envelhecimento, está o processo de confecção de uma colostomia definido pela Associação Brasileira de Ostomizados (ABRASO) como a cirurgia da vida. Sua necessidade advém de processos inflamatórios agudos, crônicos e hereditários, incontinência anal, traumas abdominais e perineais, doenças congênitas e oncológicas, concluindo que as causas em sua maioria são as doenças crônicas 5 .

Uma ostomia consiste na exteriorização de certa porção de uma víscera oca do corpo com destino a uma bolsa coletora externa ${ }^{6}$. Por essa abertura ocorre a eliminação das fezes, urina e/ou outros fluidos corpóreos ${ }^{4}$. É um processo que salva vidas, porém pode se mostrar traumático por exigir uma mudança cotidiana para o indivíduo e aqueles que convivem com ele, pois o ostoma, por suas características, não poderá ser controlado voluntariamente. Considerandose os tipos de ostomia, a colostomia é a mais frequente e caracteriza-se pela exteriorização do cólon através da parede abdominal com o objetivo da eliminação fecal ${ }^{7}$.

\section{O Idoso e a estomia}

De acordo com a etiologia da doença, o cirurgião indica a realização de um estoma temporário ou definitivo. Os temporários são aqueles que posteriormente permitirão o restabelecimento da continuidade do trato intestinal, desde que se tenha resolvido o problema que levou ao procedimento. Os definitivos ou permanentes são realizados quando não existe a possibilidade de restabelecer o trânsito intestinal, uma vez que o segmento distal do intestino foi extirpado ${ }^{8}$.

Além das mudanças de caráter fisiológico, descrevese que mudanças na vida destas pessoas podem gerar consequências psicológicas e sociais (...) Conviver com outro "membro" em seu corpo poderá causar medos, constrangimentos e dúvidas a estas pessoas 9 .

Ainda no que se trata de modificação da estrutura corpórea e cotidiana, afirma-se que, nesta vivência, atividades que parecem simples como vestir-se, tomar banho, cozinhar, ou andar de ônibus são extremamente valorizadas ${ }^{10}$. Isto requer do enfermeiro atenção aos aspectos sociais desta mudança.

Diante do processo de envelhecimento, o ser humano trilha diversas mudanças que necessitam de um novo olhar na ótica da complexidade, ou seja, em que haja uma reabilitação gradativa com vistas à sua adaptação à nova condição - idoso com estomia. Os idosos estomizados, objetos deste estudo, são sujeitos com potencialidades, porém apresentam maior fragilidade, incertezas e medos não exteriorizados, devido às mudanças que ocorrem em seu corpo e frente às novas responsabilidades que devem assumir com a manutenção de um novo dispositivo ${ }^{11,12}$.

A prática de enfermagem baseada em evidências permite a implementação de intervenções efetivas na assistência à saúde do idoso estomizado. Nesse sentido, o presente estudo procurou responder a seguinte questão: quais as evidências científicas de enfermagem acerca das pessoas idosas estomizadas?

O objetivo geral deste estudo foi analisar a atenção a idosos estomizados e como objetivos específicos pretendeuse analisar as principais dificuldades vivenciadas por esses sujeitos e descrever as estratégias de enfermagem frente às dificuldades vivenciadas por eles.

Justifica-se pelo crescimento do número de pessoas idosas, que de fato resultará em um maior número absoluto de idosos em condições de vulnerabilidade, apesar de haver redução no número de adoecimento desta faixa etária devido às ações de prevenção de doenças e melhoria nas práticas assistenciais ${ }^{13}$. Esse quadro resulta no aumento da demanda para o cuidado e na enfermagem gerontogeriátrica, na qual a principal meta no cuidado é a manutenção da autonomia e da capacidade funcional do idoso.

0 cuidado ao idoso estomizado pode ser visto como um desafio devido às experiências vivenciadas por ele que são diversas e modificam-se no decorrer do tempo, mais do que se comparadas aos mais jovens.

\section{METODOLOGIA}

Trata-se de um estudo exploratório e descritivo, realizado por meio de revisão integrativa da literatura. Este método de investigação viabiliza a busca, avaliação 
crítica e a síntese das evidências disponíveis sobre o tema. Para que seja realizada a revisão integrativa de literatura é necessário que o pesquisador siga as seis etapas inerentes a esse método ${ }^{14}$.

$\mathrm{Na}$ primeira etapa foi realizada a escolha da temática de pesquisa e a delimitação da questão que norteou a revisão integrativa. Em seguida, houve o estabelecimento dos critérios de inclusão e de exclusão dos estudos e a busca na literatura ${ }^{14}$.

Foram considerados os seguintes critérios de inclusão: artigos que abordassem pessoas acima de 60 anos de idade; artigos publicados em português, inglês ou espanhol; que fossem localizáveis com os descritores "Colostomia", "Idoso" e "enfermagem"; artigos em que pelo menos um dos autores possuíssem graduação em enfermagem, recorte temporal de dez anos (2005-2015), considerando a aprovação das portarias que garantem direitos aos portadores de Estomias. Como critério de exclusão, optouse pela eliminação dos artigos que não estivessem em conformidade com os objetivos do estudo e aqueles de acesso indisponível.

Os dados sobre o tema foram levantados nas bases de dados: Literatura Latino Americana e do Caribe em Ciências da Saúde (LILACS), Base de Dados de enfermagem (BDENF), MEDLINE (Literatura Internacional em Ciências da Saúde), IBECS (Instituto Brasileiro De Ensino Em Ciências Da Saúde) e a Biblioteca da SciELO (CientificElectronic Library Online).

A busca dos artigos publicados deu-se por meio da associação em duplas e um trio dos descritores Colostomia, Idoso, Enfermagem, conforme sugerido pelo portal de Descritores em Ciências da Saúde (DeCS), com o uso do operador booleano AND. Também foram utilizados os respectivos correspondentes dos descritores supra nos idiomas inglês e espanhol.

$\mathrm{Na}$ terceira etapa ocorreu a categorização dos estudos com objetivo de agrupá-los de maneira clara para facilitar a compreensão dos tópicos abordados ${ }^{14}$. Para organizar os estudos foi confeccionada uma tabela no software Microsoft Office Excel 2010 com as seguintes variáveis: ano de publicação, base de dados, periódico, título do artigo e principais resultados (síntese).

Na quarta etapa foi realizada a avaliação dos estudos incluídos na revisão integrativa. Os estudos foram analisados criticamente para que fosse possível explicitar resultados semelhantes ou conflitantes entre eles. A interpretação dos resultados, quinta etapa deste estudo, foi composta pela discussão dos resultados da pesquisa, o que exigiu a comparação dos estudos realizados com o conhecimento teórico.

Na sexta e última etapa foi apresentada a revisão/ síntese do conhecimento. Essa etapa consiste na elaboração do documento que deve conter as etapas percorridas pelo revisor para o alcance dos resultados. Cabe ressaltar que a quinta e sexta etapas foram cumpridas ao longo do corpo textual. A coleta de dados foi realizada no mês de dezembro de 2015 e janeiro de 2016.

Entre as produções encontradas foram selecionadas apenas aquelas que passaram pelo crivo dos critérios de inclusão e exclusão deste estudo.

\section{RESULTADOS}

Feitas as associações dos descritores nas bases de dados, foram encontrados (E) 100 artigos e selecionados (S) 18, sendo na LILACS 20 (E) e 11 (S), MEDLINE 66 (E) e 01 (S), BDENF 11 (E) e 04 (S), IBECS 02 (E) e 1(S) e, SciELO 01(E) e 1(S), conforme quadro 1.

Quadro 1. Quantitativo (n) dos artigos encontrados (E) e selecionados (S) após revisão integrativa por base de dados. Niterói - RJ, 2016.

\begin{tabular}{|l|c|c|}
\hline \multirow{2}{*}{ BASE DE DADOS } & \multicolumn{2}{|c|}{ DESCRITORES } \\
\cline { 2 - 3 } & $\begin{array}{c}\text { "IDOSO" and "COLOSTOMIA" and } \\
\text { "ENFERMAGEM" }\end{array}$ \\
\cline { 2 - 3 } & $\mathbf{E}$ & $\mathbf{S}$ \\
\hline LILACS & 20 & 11 \\
\hline BDENF & 11 & 04 \\
\hline MEDLINE & 66 & 01 \\
\hline IBECS & 02 & 01 \\
\hline SCIELO & 01 & 01 \\
\hline SOMATÓRIO POR \\
TERMO
\end{tabular}

No quadro 2 encontra-se a síntese dos artigos selecionados para a composição da discussão. Observa-se que existe uma predominância de artigos publicados em revistas de enfermagem, não especializadas no assunto.

Quanto ao ano de publicação, ao longo desses dez anos, não houve um aumento na produção acerca do assunto abordado. Não foram encontradas publicações sobre a temática nos anos de 2006 a 2007; em 2005, 01 (5,56\%) artigo; em 2008 e 2009, 2(11,11\%) e 02 (11,11\%) artigos, seguido por 2010 e 2011 com 01(5,56\% e 5,56\%) artigo cada um. 0 maior quantitativo de artigos publicados foi registrado nos anos de 2012 e 2014 - 05 (27,78\%) artigos e 03 (16,67\%) artigos -, respectivamente; em 2013 foram produzidos 02 (11,11\%) artigos e em 2015, 01 (5,56\%) artigo somente foi encontrado.

$\mathrm{Na}$ região Sudeste do país a pesquisa com idosos ostomizados se apresenta em maior número, sendo 08 $(44,44 \%)$ artigos encontrados; seguida pela região Sul com $06(27,78 \%)$ artigos concentrados na mesma área, inclusive. Na região Nordeste foram produzidos 02 (11,11\%) artigos e na região Centro-oeste apenas 01. Foram encontrados 02 (11,11\%) artigos internacionais, sendo 01 (5,56\%) da Espanha e 01 (5,56\%) EUA.

Identificaram-se os locais da pesquisa com a seguinte distribuição: Rio Grande 05 (27,78\%) e Florianópolis 01(5,56\%) artigos. São Paulo, 04 (22,22\%); Rio de Janeiro, 02(11,11\%) e Minas Gerais 02 (11,11\%) artigos. No Piauí, 01 (5,56\%); 01 (5,56\%) no Ceará; 01 (5,56\%) em Brasília e 02 (11,11\%) fora do Brasil. 
Quadro 2. Quadro-síntese dos principais resultados por ano, base de dados, revista, título do artigo e resultados.

\begin{tabular}{|c|c|c|c|}
\hline $\begin{array}{c}\text { Ano } \\
\text { Base de dados }\end{array}$ & Revista & Título do artigo & Resultados e nível de evidência \\
\hline 2015 IBECS & Metas enferm & $\begin{array}{l}\text { Calidad de vida en los pacientes } \\
\text { ostomizados un año despues } \\
\text { de lacirugía }\end{array}$ & $\begin{array}{l}\text { A fim de melhorar a aumentar a } \\
\text { qualidade de vida dos pacientes } \\
\text { ostomizados, profissionais e práticas } \\
\text { de especialistas são necessários, } \\
\text { este acompanhamento irá prevenir o } \\
\text { desenvolvimento de complicações do } \\
\text { estoma.2C }\end{array}$ \\
\hline 2014 BDENF & REAS (Internet) & $\begin{array}{l}\text { Aspectos clínicos e } \\
\text { epidemiológicos de } \\
\text { estomizados intestinais de um } \\
\text { município de Minas Gerais }\end{array}$ & $\begin{array}{l}\text { O enfermeiro pode exercer influência } \\
\text { sobre a adaptação à nova condição de } \\
\text { vida e sua reinserção social por meio do } \\
\text { cuidado oferecido a esse paciente e da } \\
\text { educação em saúde. } 2 \text { C }\end{array}$ \\
\hline 2014 MEDLINE & Supportcarecancer & $\begin{array}{l}\text { Caregivers as Healthcare } \\
\text { Managers: Health Management } \\
\text { Activities, Needs and Caregiving } \\
\text { Relationships for Colorectal } \\
\text { Cancer Survivors with Ostomies }\end{array}$ & $\begin{array}{l}\text { Os profissionais de saúde envolvidos no } \\
\text { ensino de cuidadores geralmente têm } \\
\text { como foco as necessidades físicas de } \\
\text { sobrevivência.2C }\end{array}$ \\
\hline 2014 LILACS & Rev.BrasEnferm & $\begin{array}{l}\text { Ações ecossistêmicas e } \\
\text { gerontotecnológicas no cuidado } \\
\text { de enfermagem complexo ao } \\
\text { idoso estomizado. }\end{array}$ & $\begin{array}{l}\text { Entende-se por ações ecossistêmicas, } \\
\text { a construção de um ambiente } \\
\text { terapêutico, garantia de acesso físico } \\
\text { e adaptações ambientais. Por ações } \\
\text { gerontotecnológicas, o processo } \\
\text { educativo com cartilhas ou manuais } \\
\text { acerca das orientações para cuidados } \\
\text { com estomias. 2C }\end{array}$ \\
\hline 2013 LILACS & REME & $\begin{array}{l}\text { A estomia mudando a vida: } \\
\text { enfrentar para viver }\end{array}$ & $\begin{array}{l}\text { O enfermeiro, devido à proximidade } \\
\text { gerada pelo maior contato com o } \\
\text { paciente, pode auxiliá-lo a descobrir as } \\
\text { estratégias disponíveis para o melhor } \\
\text { enfrentamento da condição de ser } \\
\text { estomizado. } 2 \text { C }\end{array}$ \\
\hline 2013 BDENF & RENE & $\begin{array}{l}\text { Prática de autocuidado de } \\
\text { estomizados: contribuições da } \\
\text { teoria de orem }\end{array}$ & $\begin{array}{l}\text { A complexidade da assistência de } \\
\text { enfermagem prestada ao estomizado } \\
\text { remete à imprescindível compreensão } \\
\text { das modificações que ocorrem em } \\
\text { sua vida e como ele vivencia todo } \\
\text { processo.2C }\end{array}$ \\
\hline 2012 LILACS & Cad. Saúde Colet. & $\begin{array}{l}\text { O perfil dos pacientes } \\
\text { estomizados com diagnóstico } \\
\text { primário de câncer de reto } \\
\text { em acompanhamento em } \\
\text { programa de reabilitação. }\end{array}$ & $\begin{array}{l}\text { A base de dados informatizada } \\
\text { caracteriza-se como uma preciosa } \\
\text { ferramenta gerencial, pois oferece } \\
\text { agilidade no acesso aos dados } \\
\text { fundamentais ao planejamento dos } \\
\text { rumos da assistência no programa em } \\
\text { foco.2C }\end{array}$ \\
\hline 2012 SciELO & Texto Cont. Enferm & $\begin{array}{l}\text { Plano de cuidados } \\
\text { compartilhado junto a clientes } \\
\text { estomizados: a pedagogia } \\
\text { Freireana e suas contribuições } \\
\text { à prática educativa da } \\
\text { enfermagem. }\end{array}$ & $\begin{array}{l}\text { A pedagogia freireana revelou-se } \\
\text { como um instrumento facilitador } \\
\text { naaprendizagem de clientes } \\
\text { estomizados no âmbito } \\
\text { de uma proposta educativa do } \\
\text { cuidado de enfermagemno contexto } \\
\text { ambulatorial.2C }\end{array}$ \\
\hline 2012 LILACS & Rev Bras Enferm & $\begin{array}{l}\text { Ser humano idoso estomizado } \\
\text { e ambientes de cuidado: } \\
\text { Reflexão sob a ótica da } \\
\text { complexidade }\end{array}$ & $\begin{array}{l}\text { Os ambientes de cuidado refletem o } \\
\text { contexto de fragilidade e necessidade } \\
\text { de cuidado do idoso com estomia, em } \\
\text { meio a um processo circular e recursivo. } \\
\text { E necessário, assim, um conhecimento } \\
\text { técnico-cientifico adequado e } \\
\text { humanizado/ampliado do enfermeiro.20 }\end{array}$ \\
\hline 2012 LILACS & Rev Gaúcha Enferm. & $\begin{array}{l}\text { Gerontotecnologia educativa } \\
\text { voltada ao idoso estomizado à } \\
\text { luz da complexidade. }\end{array}$ & $\begin{array}{l}\text { A gerontotecnologia é vista como um } \\
\text { instrumento que serve como tecnologia } \\
\text { contributiva para o cuidado à saúde do } \\
\text { idoso estomizado.2C }\end{array}$ \\
\hline
\end{tabular}


Quadro 2. Continuação

\begin{tabular}{|c|c|c|c|}
\hline $\begin{array}{c}\text { Ano } \\
\text { Base de dados }\end{array}$ & Revista & Título do artigo & Resultados e nível de evidência \\
\hline 2012 BDENF & $\begin{array}{l}\text { Rev. Latino-Am. } \\
\text { Enfermagem }\end{array}$ & $\begin{array}{l}\text { Associação dos fatores } \\
\text { sociodemográficos e clínicos } \\
\text { à qualidade de vida dos } \\
\text { estomizados. }\end{array}$ & $\begin{array}{l}\text { O estoma e o câncer, para os sujeitos } \\
\text { pesquisados, não representam } \\
\text { impacto negativo em suas vidas, desde } \\
\text { que sejam assistidos de maneira } \\
\text { humanizada e sistematizada pelos } \\
\text { enfermeiros.2C }\end{array}$ \\
\hline 2011 LILACS & $\begin{array}{l}\text { Revista Brasileira de } \\
\text { Enfermagem }\end{array}$ & $\begin{array}{l}\text { Autoimagem de clientes com } \\
\text { colostomia em relação à bolsa } \\
\text { coletora. }\end{array}$ & $\begin{array}{l}\text { A proposta de estímulo ao autocuidado } \\
\text { tem sido descrita como uma alternativa } \\
\text { terapêutica que possibilita que o } \\
\text { paciente participe ativamente do } \\
\text { seu tratamento, estimulando a } \\
\text { responsabilidade na continuidade do } \\
\text { cuidado pós alta hospitalar.2C }\end{array}$ \\
\hline 2010 LILACS & Rev. enferm. UERJ & $\begin{array}{l}\text { A percepção de si como } \\
\text { ser-estomizado: um estudo } \\
\text { Fenomenológico }\end{array}$ & $\begin{array}{l}\text { O ser estomizado é compreendido na } \\
\text { sua singularidade como expressividade } \\
\text { corporal que revela a sua vivência } \\
\text { existencial e experiência na perspectiva } \\
\text { de seu processo de adoecimento.2C }\end{array}$ \\
\hline 2009 BDENF & $\begin{array}{l}\text { Rev. Latino-Am. } \\
\text { Enfermagem }\end{array}$ & $\begin{array}{l}\text { Os sentidos de ser homem } \\
\text { com estoma intestinal por } \\
\text { câncer colorretal: Uma } \\
\text { abordagem na antropologia das } \\
\text { masculinidades }\end{array}$ & $\begin{array}{l}\text { A enfermagem tem papel crucial no } \\
\text { reconhecimento das subjetividades } \\
\text { masculinas dos homens com colostomia, } \\
\text { construídas no convívio sociocultural.2C }\end{array}$ \\
\hline 2009 LILACS & Rev. Enferm. UERJ & $\begin{array}{l}\text { O cuidado à pessoa portadora } \\
\text { de estomia: o papel do Familiar } \\
\text { cuidador }\end{array}$ & $\begin{array}{l}\text { O cuidado afetivo revela-se como } \\
\text { um suporte no sentido de auxiliar o } \\
\text { portador de estomia a melhorar sua } \\
\text { autoestima e autoimagem; cada família } \\
\text { é única e passa por esse processo de } \\
\text { maneira própria. } 2 \text { C }\end{array}$ \\
\hline 2008 LILACS & $\begin{array}{l}\text { Rev. Latino-Am. } \\
\text { Enfermagem }\end{array}$ & $\begin{array}{l}\text { Estratégias de enfrentamento } \\
\text { (coping) de pessoas } \\
\text { ostomizadas. }\end{array}$ & $\begin{array}{l}\text { Espera-se a reflexão e a utilização dos } \\
\text { conhecimentos pelos profissionais } \\
\text { assistenciais e de ensino, que } \\
\text { possam identificar as estratégias de } \\
\text { enfrentamento à luz do referencial de } \\
\text { coping, utilizadas pelos ostomizados; } 2 \text { C }\end{array}$ \\
\hline 2008 LILACS & $\begin{array}{l}\text { Revista da Rede de } \\
\text { Enfermagem do Nordeste }\end{array}$ & $\begin{array}{l}\text { O cuidado de enfermagem à } \\
\text { pessoa idosa estomizada na } \\
\text { perspectiva da complexidade }\end{array}$ & $\begin{array}{l}\text { Os cuidados de enfermagem carecem } \\
\text { de reformulação e reconstrução do } \\
\text { entendimento acerca da estomia como } \\
\text { parte de uma nova imagem corporal. C4 }\end{array}$ \\
\hline 2005 LILACS & Texto Contexto Enferm & $\begin{array}{l}\text { A trajetória do grupo de apoio à } \\
\text { pessoa ostomizada: projetando } \\
\text { Ações em saúde e } \\
\text { compartilhando vivências e } \\
\text { saberes }\end{array}$ & $\begin{array}{l}\text { A articulação das ações de saúde } \\
\text { em parcerias interinstitucionais, } \\
\text { interdisciplinares e interpessoais } \\
\text { fomenta a aprendizagem, a produção e } \\
\text { socialização do conhecimento de todos } \\
\text { os envolvidos.2C }\end{array}$ \\
\hline
\end{tabular}

Sobre o tipo e objetivos, dos dezoito estudos, 13 (72,22\%) foram de abordagem qualitativa, 01 (5,56\%) multicêntrico e 03 (16,67\%) quantitativos. Foram encontrados 14 (77,78\%) artigos descritivos, 02 (11,11\%) etnográficos, 01 (5,56\%) observacional e 01 (5,56\%) convergente assistencial.

Nos 18 artigos selecionados, quanto à formação das autoras, verificou-se que em todos eles participaram enfermeiras (100\%) e em 15 (83,3\%) artigos foram encontradas exclusivamente enfermeiras como autoras. Participaram 03 (16,67\%) médicos, 03 (16,67\%) acadêmicos de enfermagem, 01 (5,56\%) terapeuta ocupacional, 01 (5,56\%) psicóloga e 01 (5,56\%) administradora.
Foi observada a diferença de termos utilizada entre os artigos ao se tratar da ostomia. Em 12 (66,67\%) dos estudos os autores referem o tema como estomia/estomização e em 06 (33,3\%), ostomia/ostomização. No presente estudo, optou-se em utilizar os termos "estoma/confecção de um estoma" (de maneira genérica) ou "colostomia/confecção de uma colostomia" (de maneira específica), conforme o preconizado pela Associação Brasileira de Estomaterapia (SOBEST).

Nota-se, portanto, em relação ao nível de evidência, que a maioria dos artigos possui classificação intermediária (2C) pelos seus resultados apresentarem relativo ou baixo 
potencial de refletir na prática clínica, logo, a necessidade de que mais estudos que venham contribuir nesta perspectiva.

Tabela 1. Número de artigos publicados acerca da colostomia em idosos, com base nas produções científicas nacionais e internacionais, por periódico - 2005 a 2015.

\begin{tabular}{l|c}
\hline \multicolumn{1}{c|}{ Periódico } & $\begin{array}{c}\text { Número de artigos } \\
\text { publicados }\end{array}$ \\
\hline Metas de Enfermería & 01 \\
\hline Revista de Enfermagem e Atenção à Saúde & 01 \\
\hline Latino Americana de Enfermagem & 03 \\
\hline Cadernos de Saúde Coletiva & 01 \\
\hline Texto \& Contexto Enfermagem & 02 \\
\hline Revista Brasileira de Enfermagem & 02 \\
\hline Revista Gaúcha de Enfermagem & 01 \\
\hline Revista Mineira de Enfermagem & 01 \\
\hline Revista de Enfermagem UERJ & 02 \\
\hline Revista da Rede de Enfermagem do & 02 \\
Nordeste & 01 \\
\hline Revista Brasileira de Enfermagem de & 01 \\
\hline Brasília & 18 \\
\hline SupportCareCâncer & Total \\
\hline
\end{tabular}

Fonte: Elaboração própria

\section{DISCUSSÃO}

Após análise dos referidos artigos foram criadas duas categorias: dificuldades enfrentadas pela pessoa idosa colostomizada e Estratégias de enfermagem no enfrentamento das dificuldades vivenciadas pelos idosos colostomizados.

\section{Dificuldades enfrentadas pela pessoa idosa colostomizada}

O idoso colostomizado demanda ações de cuidados peculiares ao seu novo estado de saúde, tendo como uma das principais dificuldades a aceitação de sua nova condição, que a princípio é vista como debilitante para o convívio social e pode resultar em dependência para o cuidado consigo mesmo ${ }^{15,16}$.

A colostomia pode incidir como um marcador temporal na vida dos idosos, apresentando o limiar entre o antes e o após de uma vida com novas demandas de cuidado e assistência, sendo importante a promoção do autocuidado que poderá ser praticado na medida em que o idoso aprende a conviver com a bolsa de colostomia ${ }^{15,17}$.

A pessoa idosa poderá experienciar uma situação de envelhecimento somada a confecção de um estoma, que pode resultar em sentimentos negativos e depreciação da própria imagem, além de alterações no humor. Esta situação poderá agravar ainda mais o estado do indivíduo, que poderá negar o tratamento e perder o gosto pela vontade de continuar lutando pela vida15,18,19,20.

O processo de aceitação pode ser longo e duradouro quando o idoso desconhece aquele novo mundo no qual passa estar inserido. $\mathrm{O}$ idoso começa a viver um estado de ansiedade, pois desconhece a colostomia, e somente após entender essa nova realidade será capaz de criar novos meios para se readaptar ao seu cotidiano social ${ }^{21,22,16,9}$.

O dano corporal provocado pela confecção do estoma, como a violação do corpo para higienização e a perda da função regulatória nas eliminações intestinal/urinária, irá diminuir a qualidade de vida do idoso. Esse indivíduo necessitará rever hábitos diários, como a nutrição e manipulação do estoma 15, 18-19,23-25, 9 .

A realização dos exames de colonoscopia e toque retal estão entre os exemplos do rompimento da integridade física e psicossocial do idoso que detém diagnóstico favorável à condição de se tornar colostomizado. Esses danos são relatados no sexo masculino como um rompimento da sua condição de intocável, resistente e forte, que pode ser agravada pelo medo destes sujeitos na manutenção da atividade sexual ${ }^{18,20}$.

Salienta-se que as modificações advindas com a colostomia têm impacto significativo na manutenção das atividades sexuais dos pacientes. Em muitos casos, os indivíduos não conseguem manter a performance anterior ao procedimento, entrando em diversos conflitos psicossociais esse problema pode incidir em destruição do espaço familiar e agravar as dificuldades da pessoa idosa colostomizada ${ }^{19,26}$.

Uma das formas que os idosos mencionam para fugir do estigma e melhorar sua relação social é ocultar a bolsa de colostomia com o uso de roupas que não permitam a visualização do seu estado de saúde e, dessa forma, possibilite tentar viver como as demais pessoas, sentindo-se "normal"23. Nesse cenário sombrio de queda da autoestima a família poderá funcionar como fonte de apoio frente essa nova condição. Nesse laço familiar, ações positivas, irão implicar na diminuição do medo e redução do sofrimento e dor, que possivelmente permearão o idoso colostomizado. 0 núcleo familiar é fundamental para que o idoso não venha a isolar-se socialmente $27,22,24$.

Em muitos casos a própria organização do sistema de atendimento nos serviços de saúde se mostra como fator que dificulta a vida dos idosos com essa demanda de cuidado. Muitos pacientes caminham um longo percurso permeado por burocracia e dificuldades de recursos no atendimento, tais como inúmeros encaminhamentos entre diferentes instituições, longos agendamentos, realização de exames, afastamento das atividades laborais, recursos econômicos ${ }^{18}$.

A organização do sistema é relevante, mesmo após a conquista do atendimento e a realização da estomia pois devido à condição financeira dos idosos há o receio de não conseguirem gratuitamente a bolsa de colostomia 
conforme necessidade posterior. Assim, há uma insegurança no atendimento oferecido pelo sistema público de saúde ${ }^{24}$.

\section{Estratégias de enfermagem no enfrentamento das dificuldades vivenciadas pelos idosos colostomizados}

A educação em saúde é uma das ferramentas mais determinantes no enfrentamento das dificuldades encontradas pelos idosos colostomizados, uma vez que através do diálogo se permite o compartilhar de saberes e práticas acerca dessa nova condição de vida no idoso, além de promover o autocuidado, possibilitando a melhora da qualidade de vida e inserção nos diferentes grupos sociais $29,17,11,27,23,16,9,25,19-20$

O idoso que vivencia a condição de ser estomizado necessita de uma abordagem integrale de acordo com os princípios universais de cuidado a saúde, uma vez que é um sujeito com opiniões próprias, contexto sociocultural único e vivências singulares, portanto com uma autenticidade única ${ }^{17,30-31,22,16}$.

A notícia da confecção de uma colostomia ao idoso dever ser uma das principais abordagens na qual o profissional de saúde deve estar preparado. O enfermeiro poderá ajudar ao idoso informando estratégias de cuidado a sua nova condição e oferecendo suporte para esse paciente, possibilitando que este sinta-se seguro e tenham vontade de superar a condição debilitante na qual se encontra ${ }^{17,26,9}$.

O enfermeiro pode ajudar o idoso com suas demandas de cuidado, uma vez que além de dar suporte poderá ser um profissional com escuta atenta, que permitirá que esse idoso se sinta seguro e motivado a continuar lutando pela vida e não caia numa condição de isolamento ou segregação social por causa da condição de saúde ${ }^{17}$.

A família também se apresenta como um grande elemento de enfrentamento do problema e pode facilitar o processo de adaptação do estoma, além de estimular o idoso para melhorar sua qualidade de vida e gerar dados aos enfermeiros para diferentes estratégias de enfrentamento e superação. Uma vez que ninguém conhece mais esse idoso do que seu próprio familiar ${ }^{17-18,11,23-24,28,25}$.

A força advinda dos familiares e amigos dos idosos pode ajudar o paciente a vencer os sentimentos negativos e falta de esperança. 0 enfermeiro deverá nesse momento praticar um cuidado que oportunize esse processo e, portanto, que permitia a reabilitação psicossocial do idoso mesmo antes do pré-operatório até sua posterior reinserção social ${ }^{19,26,28,9,20}$.

A abordagem intersetorial e multidisciplinar permite a propagação de conhecimentos e atitudes positivas para enfrentar as diversas dificuldades na colostomia, além de permitir aos idosos dividir suas experiências com outros sujeitos em situação semelhante e, dessa forma, sentirem mais seguros com a condição na qual se encontram ${ }^{19,22,28-29}$.

$\mathrm{Na}$ literatura, sugere-se a importância do grupo de estomizados, onde idosos com características e demandas de saúde semelhantes são capazes juntos, de reconstruir seus significados acerca das suas condições e demandas de saúde, a fim de aperfeiçoar as estratégias de enfrentamento e conseguirem rapidamente sanar dúvidas acerca da estomia. Assim, o enfermeiro poderia ser um profissional facilitador dessa estratégia e permitir no rol dos pacientes atendidos uma nova abordagem do cuidar e fazer saúde ${ }^{17,22,9,19-20}$.

\section{CONCLUSÃO}

As evidências da pesquisa explicitam que estão entre as dificuldades enfrentadas pela pessoa idosa estomizada, o desconhecimento, a ansiedade, o medo, as alterações nutricionais e de lazer, as alterações da imagem corporal, o rompimento da integridade física e psicossocial, a manutenção da atividade sexual e recursos financeiros. Desta forma, estas pessoas demandam um cuidado voltado às peculiaridades inerentes ao processo de envelhecimento. Os cuidados com a colostomia exigem que seu portador possua conhecimento a fim de evitar problemas de cicatrização, infecções e até mesmo odores provenientes da bolsa, um dos problemas que mais afetam as pessoas idosas estomizadas responsáveis pelo constrangimento. Desta forma, é necessário que a pessoa idosa portadora de ostomia tenha as informações que colaborem para o processo de compreensão de sua nova condição, visto que se trata de um procedimento invasivo, doloroso físico e emocionalmente.

A educação em saúde da pessoa idosa colostomizada se mostrou como estratégia mais eficaz no processo adaptativo deste procedimento e não apenas para o seu portador, mas também para a família, que em sua maioria, tem um membro como cuidador. 0 cuidado de enfermagem tem como objetivo promover o autocuidado e a autonomia da pessoa idosa e dedica-se à escuta atenta e à motivação para que esta clientela não venha a isolar-se socialmente.

Visando evitar o isolamento, os grupos de ostomizados são sugeridos por reunir pessoas em condições semelhantes e que buscam aperfeiçoamento de suas estratégias de enfrentamento, pois é um espaço de troca de conhecimentos, onde o enfermeiro torna-se mediador, um profissional facilitador dessa estratégia, e permite a inserção de uma nova abordagem do cuidar, oriunda de equipes multidisciplinares para um suporte biológico, psicológico e social.

Tendo em vista as dificuldades e estratégias de enfrentamento, conclui-se que o enfermeiro gerontológo possui conhecimentos que possibilitam que a qualidade de vida se perpetue nesse segmento populacional, minimizando os danos à medida que conhece a condição e limites da pessoa idosa e percebe a importância do autocuidado e manutenção da sua qualidade de vida.

Considerando que a atenção às pessoas ostomizadas exige estrutura especializada e profissionais capacitados, conforme a Portaria $N^{\circ}$ 400, de 16 de novembro de 2009, conclui-se que existe a necessidade de capacitação dos 
profissionais envolvidos na gestão dos serviços de saúde, visto que nesta busca não foram encontrados artigos que relatassem conhecimento acerca desta portaria. Além disso, o estudo permitiu, por meio da busca na literatura, uma avaliação crítica e síntese das evidências atuais disponíveis sobre idosos estomizados, com a implementação de intervenções efetivas na assistência à saúde dessa clientela e identificação de lacunas para direcionar o desenvolvimento de futuras pesquisas.

0 estudo traz como importante consideração a necessidade da participação ativa do profissional de enfermagem atrelada à especialidade em gerontologia como complemento ao cuidado da pessoa idosa estomizada.

Por fim, destaca-se a necessidade de realização de pesquisas com metodologias de maior nível de evidência sobre a atenção aos idosos estomizados, por enfermeiros, fortalecendo o conhecimento quanto a esta temática baseada em evidências científicas que conduzam práticas mais especializadas.

\section{REFERÊNCIAS}

1. Oliveira, LPBA, Menezes RMP. Representações de fragilidade para idosos no contexto da estratégia da saúde da família. Texto \& Contexto Enfermagem [internet]. 2011 [acesso em 02 abr 2016]; 20(2): 301-9. Disponível em: http://www. scielo.br/pdf/tce/v20n2/a12v20n2.

2. Brasileiro de Geografia e Estatística [Internet]. Projeção da População do Brasil por sexo e idade:1980-2050-Revisão 2008 [acesso em 02 abril 2016]. Disponível em: http://www.ibge.gov. br/home/estatistica/populacao/projecao_da_populacao/2008.

3. Instituto Brasileiro de Geografia e Estatística [Internet]. Censo Demográfico 2010 [acesso em 02 abril 2016]. Disponível em: http://www.ibge.gov.br/home/estatistica/populacao/censo2010/ default.shtm.

4. Ministério da Saúde (Brasil) [Internet]. Secretaria de Atenção à Saúde, Departamento de Atenção Básica. Cadernos de Atenção Básica: estratégias para o cuidado da pessoa com doença crônica. Brasília: Ministério da Saúde; 2014. (35) [acesso em 02 abr 2016]. Disponível em: http://bvsms. saude.gov.br/bvs/publicacoes/estrategias_cuidado_pessoa_ doenca_cronica_cab35.pdf.

5. Associação Brasileira de Ostomizados [Internet]. Quantitativo aproximado de pessoas ostomizadas no Brasil 2007 [acesso em 02 abril 2016]. Disponível em: http://www.abraso.org.br/ estatistica_ostomizados.htm.

6. Oliveira G, Maritan CVC, Mantovanelli C, Ramalheiro GR, Gavilhia TCA, Paulo AAD. Impacto da estomia: sentimentos e habilidades desenvolvidos frente à nova condição de vida. Rev Estima [Internet]. 2010 [acesso em 02 abr 2016]; 8(1): 19-25. Disponível em: http://www.revistaestima.com. br/index.php?option=com_content \&view=article\&id $\quad=19$ : artigo-original-2 \& catid=14: edicao-81\&Itemid=25.

7. Stumm EMF, Oliveira ERA, Kirschner RM. Perfil de pacientes ostomizados. Scientia Medica [Internet]. 2008 [acesso em 02 abr 2016]; 18(1): 26-30. Disponível em: http:// revistaseletronicas.pucrs.br/ojs/index.php/scientiamedica/ article/viewFile/2552/7850.
8. Santos VLCG. Cuidando do ostomizado: análise da trajetória no ensino, pesquisa e extensão [tese] [Internet]. São Paulo: Universidade de São Paulo; 2006. [Acesso em 02 abr. 2016]. Disponível em: http://www.teses.usp.br/teses/disponiveis/ livredocencia/7/tde-15092006-145018/publico/ teseformatacaofinal1.pdf.

9. Barbosa MH, Poggetto MTD, Barrichello E, Cunha DF, Silva R, Alves PIC, Luiz RB. Aspectos clínicos e epidemiológicos de estomizados intestinais de um município de Minas Gerais. Rev. Enferm e Atenção à Saúde [Internet]. 2014 [acesso em 03 abr 2016]: 3(1):64-73. Disponível em: http://www. uftm.edu.br/revistaeletronica/index.php/enfer/article/ download/931/663.

10. Silva DF. O desafio do autocuidado de pacientes oncológicos ostomizados: da reflexão à ação [dissertação] [Internet]. Niterói: Universidade Federal Fluminense; 2013. [Acesso em 03 de abr 2016]. Disponível em: http://www.repositorio. uff.br/jspui/bitstream/1/1027/1/Daniela\%20Ferreira\%20 da\%20Silva.pdf.

11. Barros EJL, Santos SSC, Lunardi VL, Lunardi Filho WD. Ser humano idoso estomizado e ambientes de cuidado: reflexão sob a ótica da complexidade. Rev. Brasileira Enferm [Internet]. 2012 [acesso em 03 abr 2016]; 65(5): 844-8. Disponível em: http://www.scielo.br/pdf/reben/v65n5/19.pdf.

12. Organização Mundial de Saúde (Brasil) [Internet]. Componentes Estruturais de Ação, Relatório Mundial. Cuidados Inovadores para Condições Crônicas. Gênova: Organização Mundial de Saúde; 2002 [acesso em 03 abr 2016]. Disponível em: http://www.opas.org.br/wp-content/ uploads/2015/09/Manual_final.pdf.

13. Veras RP. Experiências e tendências internacionais de modelos de cuidado para com o idoso.Ciência \& Saúde Coletiva [Internet]. 2012 [acesso em 03 abr 2016]; 17(1): 231-8. Disponível em: http://www.scielo.br/scielo.php?script=sci_arttext \&pid=S1413$81232012000100025 \&$ lng=pt\&nrm=iso \&tlng=en.

14. Mendes KDS, Silveira RCCP, Galvão CM. Revisão integrativa: método de pesquisa para a incorporação de evidências na saúde e na enfermagem. Texto Contexto Enferm [Internet]. 2008 [acesso em 03 abr 2016]: 17(4): 758-64. Disponível em: http://www.scielo.br/scielo.php?script=sci_ arttext\&pid=S0104-07072008000400018.

15. Barros EJL, Santos SSC, Erdmann AL. Rede social de apoio às pessoas idosas estomizadas à luz da complexidade. Acta Paul. Enferm [Internet]. 2008 [acesso em 04 abr 2016]; 21(4): 595-601. Disponível em: http://www.scielo.br/scielo. php?script=sci_arttext \&pid=S0103-21002008000400010.

16. Barros EJL, Santos SSC, Gomes GC, Erdmann AL, Pelzer MT, Gautério DP. Ações ecossistêmicas e gerontotecnológicas no cuidado de enfermagem complexo ao idoso estomizado. Rev. Bras. Enferm [Internet]. 2014 [acesso em 04 abr 2016]: 67(1): 91-6. Disponível em: http://www.scielo.br/scielo. php?script=sci_arttext \&pid=S0034-71672014000100091.

17. Barnabe NC, Dell'Acqua MCQ. Estratégias de enfrentamento (coping) de pessoas ostomizadas. Rev Latino-amEnferm [Internet]. 2008 [acesso em 04 abr 2016]: 16(4). Disponível em: http://www.scielo.br/pdf/rlae/v16n4/pt_10.pdf.

18. Dázio EMR, Sonobe HM,Zago MMF. Os sentidos de ser homem com estoma intestinal por câncer colorretal: uma abordagem na antropologia das masculinidades. Rev. Latino-am 
Enfermagem [Internet]. 2009 [acesso em 04 abr 2016]; 17(5). Disponível em: http://www.scielo.br/scielo.php?pid=S0104$11692009000500011 \&$ script=sci_arttext\&tlng=pt.

19. Batista MRFF, Rocha FCV, Silva DMG, Silva Junior FJG. Autoimagem de clientes com colostomia em relação à bolsa coletora. Rev. Bras. Enferm [Internet]. 2011. [acesso em 04 abr 2016]; 64(6): 1043-7. Disponível em: http://www.scielo. br/pdf/reben/v64n6/v64n6a09.pdf.

20. McMullen CK et al. "Caregivers as healthcare managers: health management activities, needs, and caregiving relationships for colorectal cancer survivors with ostomies." SupportiveCare in Câncer [Internet] 2014 [acesso em 03 de abr. 2016] 22(9): 2401-2408. Disponível em: http://link. springer.com/article/10.1007/s00520-014-2194-3

21. Delavechia RP et al. A percepção de si como ser-estomizado: um estudo Fenomenológico. Rev. enferm. UERJ, Rio de Janeiro, 2010 abr/jun; 18(2):223-8. Acesso em 2 abr.2016; disponível em: http://www.facenf.uerj.br/v18n2/v18n2a10.pdf.

22. BarrosEIL,SantosSSC,GomesGC,ErdmannAL.Gerontotecnologia educativa voltada ao idoso estomizado à luz da complexidade. Rev. Gaúcha Enferm [Internet]. 2012 [acesso em 04 abr 2016]; 33(2): 95-101. Disponível em: http://www.scielo.br/scielo. php?script=sci_arttext \&pid=S1983-14472012000200014

23. Coelho AR, Santos FS, Poggetto MTD. A estomia mudando a vida: enfrentar para viver. Revista Mineira de Enfermagem [Internet] 2013 [acesso em 04 abr 2016]; 17(2), 258-277. Disponível em: http://www.reme.org.br/artigo/detalhes/649

24. Ministério da Saúde (Brasil) [Internet]. Instituto Nacional de Câncer José Alencar Gomes da Silva. Estimativa 2012: Incidência de Câncer no Brasil.

25. Campillo MJ. Calidad de vida enlos pacientes ostomizadosunañodespues de lacirugía." Metas de Enfermería [internet] 2015 [acesso em 03 abr 2016]; 18(8): 25-31. Disponível em: http://www.enfermeria21.com/ revistas/metas/articulo/80809/.

26. Pereira APS, Cesarino CB, Martins MRI, Pinto MH, Netinho JG. Associação dos fatores sociodemográficos e clínicos à qualidade de vida dos estomizados. Rev. Latino-Am. Enfermagem [Internet]. 2012 [acesso em 04 abr 2016]; 20(1): [08 telas]. Disponível em: http://www.scielo.br/ scielo.php?pid=S0104-11692012000100013 \& script=sci_ arttext\&tlng=pt.

27. Souza JL, Gomes GC, Barros EJL. O cuidado à pessoa portadora de estomia: o papel do familiar cuidador. Rev. Enferm. UERJ [Internet]. 2009 [acesso em 04 abr 2016]; 17(4): 550-5. Disponível em: http://www.facenf.uerj.br/v17n4/v17n4a17.pdf.

28. Menezes, LCG, Guedes MVC, Oliveira RM, Oliveira SKPD, Meneses LSTD, Castro MED. Prática de autocuidado de estomizados: contribuições da Teoria de Orem. Revista da Rede de Enfermagem do Nordeste-Rev. Rene [Internet] 2013 [acesso em 04 abr 2016]; 14(2). Disponível em: http:// www.revistarene.ufc.br/revista/index.php/revista/article/ view/235/pdf_1.

29. Martins ML, Silva RDM, Fangier A, Perugini VC, Pereira VC, D’Ávila FS, et al. A trajetória do grupo de apoio à pessoa ostomizada: projetando ações em saúdee compartilhandovivênciase saberes. Texto contexto - enferm [Internet].2005 [acesso em 04 abr 2016]; 14(4): 594-600. Disponível em: http://www.scielo.br/scielo. php?script=sci_arttext \&pid=S0104-07072005000400017.
30. Martins PAF, Alvim NAT. Plano de cuidado compartilhado junto a clientes estomizados: a pedagogia freireana e suas contribuições à prática educativa da enfermagem. Texto Contexto Enferm [Internet], 2012 [acesso em 04 abr 2016]; 21(2): 286-94. Disponível em: http://www.scielo.br/scielo. php?script=sci_arttext \&pid=S0104-07072012000200005.

31. Barros EJL, Santos SSC, Gomes GC, Erdmann AL. O cuidado de enfermagem à pessoa idosa estomizada na perspectiva da complexidade. Rev. Rede Enferm Nordeste [Internet]. 2008 [acesso em 04 abr 2016]; 9(2): 28-37. Disponível em: http:// www.redalyc.org/articulo.oa?id=324027962004. 